\title{
Implementación de sistemas de integridad como estrategia de control de la corrupción en el Gobierno Central de Chile
}

\author{
Implementing Systems of Integrity to Curb Corruption in the Chilean Central Government
}

\author{
Cristian Pliscoff Varas y Nicolás Lagos Machuca*
}

Resumen: Los gobiernos han desplegado diferentes estrategias para controlar la corrupción y reforzar los marcos éticos de las y los funcionarios públicos. Tradicionalmente se ha seguido un enfoque normativo o de la obediencia, que resalta la relevancia de las normas para evitar conductas reñidas con la ética. El gobierno chileno, luego de una importante crisis de corrupción, decidió implementar un enfoque diferente, donde se busca enfrentar esta temática a partir de un enfoque de integridad centrado en los valores de la función pública. El objetivo de este artículo es estudiar cómo se ha puesto en marcha esta estrategia, a través de un análisis cuantitativo y cualitativo, que nos lleva a presentar diferentes modos de implementación de sistemas de integridad. El artículo finaliza con reflexiones que pudiesen ser de utilidad tanto en Chile como en América Latina, para una mejor implementación de estas estrategias de prevención de la corrupción.

Palabras clave: administración pública, corrupción, integridad, ética, Chile.

Abstract: Governments have deployed different strategies for controlling corruption and to reinforce the ethical frameworks of public employees. Traditionally, a normative or "obedience" approach has been adopted, which highlights the importance of regulations to avoid unethical behaviors. The Chilean government, after an important corruption crisis, decided to implement a different approach, where the objective is to address this problem by an integrity approach centered on the values of public service. The purpose of this paper is to study how this strategy has unfolded, through a quantitative and qualitative analysis, which allows us to present different models of implementation of integrity systems. The article fi-

${ }^{*}$ Cristian Pliscoff Varas es profesor-investigador de la Universidad de Chile, Santa Lucía 240, Santiago, Chile. Tel: +56229 771 505. Correo-e: cpliscof@iap.uchile.cl. orcid: 0000-0002-6918-4587. Nicolás Lagos Machuca es investigador independiente. Tel: +569 9086 3252. Correo-e: nmlagos@gmail.com. ORCID: 0000-0001-9140-1958.

Artículo recibido el 1 de octubre de 2020 y aceptado para su publicación el 26 de marzo de 2021. 
nalizes with ideas and thoughts that could be useful, both for Chile and Latin America, for a better implementation of strategies for curving corruption.

Keywords: public administration, corruption, integrity, ethics, Chile.

\section{INTRODUCCIÓN}

- $\mathrm{n}$ abril de 2015, el Consejo Asesor Presidencial contra los Conflictos de Intereses, el Tráfico de Influencias y la Corrupción entregó a la entonces presidenta de Chile, Michelle Bachelet, un informe con más de 200 propuestas que tenían por objetivo fortalecer la probidad y la transparencia en el sector público y privado. Esta Comisión fue instalada para salir de una importante crisis institucional, donde se hacía evidente la existencia de malas prácticas en el sector público chileno (Lagos, 2019; Pliscoff, 2017; Silva, 2016). Dentro de las propuestas, se incluyó la necesidad de implementar sistemas de integridad para el sector público, cuestión que fue incorporada en la "Agenda de Probidad y Transparencia" que el gobierno de la presidenta intentó implementar durante su mandato (2014-2018). Como parte de ese esfuerzo, y luego de un trabajo de dos ańos, se anunció que 256 instituciones del sector público contaban con códigos de ética elaborados a través de una metodología participativa y bajo lineamientos centralizados emanados de la Dirección Nacional del Servicio Civil (DNSC), los cuales eran la piedra fundamental de los sistemas de integridad que debían implementarse con posterioridad.

Esta acción viene, de alguna manera, a quebrar con la tendencia de los gobiernos chilenos, y de gran parte de América Latina que, ante una crisis de corrupción, reaccionan solamente con cambios legales. Por el contrario, este esfuerzo se puede concebir como una acción concreta que va más allá de lo meramente legal, y se orienta a reforzar los marcos éticos de las y los funcionarios públicos. Al pedirle a los servicios públicos y ministerios de la administración central del Estado que desarrollaran los códigos de ética, se iniciaba un proceso tendiente a crear sistemas de integridad como herramientas efectivas para el control de la corrupción y refuerzo de los marcos éticos. A dos años de implementación de este proceso, nos parece pertinente analizar cómo este se ha llevado a cabo, ya que resulta particularmente relevante verificar si la estrategia adoptada, del tipo "topdown", ha permitido instalar estos sistemas de integridad en las organizaciones públicas chilenas. Este estudio viene a aportar a la literatura sobre implementación de sistemas de integridad en organizaciones públicas, entregando aprendizajes para otros países de la región interesados en pasar de una lucha contra la corrupción basada en la obediencia a una basada en la integridad. 


\section{PERSPECTIVAS TEÓRICAS SOBRE CORRUPCIÓN E INTEGRIDAD}

Aunque durante muchos años la corrupción fue sinónimo exclusivamente de soborno, en la actualidad existe un consenso sobre la necesidad de contar con una conceptualización más amplia que permita incorporar y medir las distintas formas en que este fenómeno se presenta (Andersson, 2017). Si bien la comprensión de la corrupción, entendida como el "abuso del poder encomendado para beneficio privado", de Transparencia Internacional (2020) es una definición ampliamente aceptada, es al mismo tiempo objeto de múltiples críticas al presentar una visión principalmente economicista de la corrupción que no considera elementos éticos, políticos y sociales (Andersson y Heywood, 2009; Heywood et al., 2017). En esta línea, Sandoval Ballesteros (2016) recalca la importancia de ver a la corrupción como un constructo general complejo con alcances sociales, institucionales y políticos. Por eso, este estudio se enfocará en una visión más amplia de este fenómeno en que, siguiendo a Andersson y Anechiarico (2019), se considera corrupción no solo como aquellos actos en beneficio propio, sino que también aquellas acciones que afectan los valores de la buena administración.

Esta línea teórica permite comprender que la ausencia de inobservancias éticas no es en sí misma una situación ideal para los gobiernos, sino que para combatir la corrupción efectivamente es necesario promover que funcionarias y funcionarios públicos se comprometan proactivamente a actuar con integridad. Es por esto que entendemos que la integridad en el sector público se ha transformado en un objetivo por alcanzar.

Este vocablo se puede utilizar de muchas maneras y sentidos. Huberts (2018) distingue ocho perspectivas para comprender este concepto, las que apuntan a definir la integridad como "la cualidad de actuar en concordancia y armonía con valores, normas y reglas de aceptación general" (p. 3). En este artículo, entendemos por integridad a "una virtud que garantiza que las acciones se basan en un marco de principios internamente consistente" (Villoria, 2014). De este modo, es posible comprender que el concepto de integridad se incorpora al ejercicio de lo público como una manera de superar los enfoques meramente deontológicos o normativos que los conceptos ética y probidad implican (Lagos y Pliscoff, 2020).

Para alcanzar niveles crecientes de integridad entre las y los funcionarios públicos, se ha optado por diferentes intervenciones e instrumentos, en lo que la literatura llama la "gestión de la ética" (ethics management) (Menzel, 2001). Dentro de los medios más utilizados para instalar la integridad en las organizaciones públicas, encontramos los códigos de ética, el liderazgo ético, las capacitaciones en 
ética, entre otros recursos. Los códigos de ética son instrumentos de gestión que permiten alinear el comportamiento de los miembros de una organización a los valores propios de la institución, donde se combinan elementos conductuales específicos, pero también reflexiones de valores y principios, orientados a la acción (Gilman, 2005). Así, promueven que las instituciones eleven el estándar ético de sus miembros y prevengan el surgimiento de casos de corrupción. Este enfoque es consistente con la visión de la corrupción como un fenómeno organizacional como bien señala Arellano-Gault (2017), ya que los instrumentos que se despliegan para enfrentar el fenómeno de la corrupción, y por ende reforzar la integridad, son esencialmente colectivos, entendiendo que la existencia de una falta a esta no es un tema solamente individual sino también de la organización.

Entendemos por sistemas de integridad al conjunto de instrumentos internos de control ético, tales como códigos de ética, capacitaciones en ética y probidad, liderazgo ético, entre otros, junto a controles externos tales como los efectuados por organismos independientes y la ciudadanía (Huberts y van Montfort, 2020b). Destacada evidencia teórica (Bautista, 2015; Huberts y Hoekstra, 2016; Jiménez, 2017) y múltiples organizaciones internacionales (INTOSAI, 2016; OCDE, 2020; PNUD, 2017) han enfatizado que la eficacia de este modo de control y reforzamiento ético está determinado por la implementación de una serie de elementos institucionales — bajo la figura de un sistema de integridad — que permita dar coherencia y sostenibilidad a los lineamientos anticorrupción a nivel nacional, local u organizacional.

Los sistemas de integridad buscan superar los temas de corrupción e incorporan prácticas que, pudiendo no ser estrictamente "corruptas", contravienen valores esenciales de la vida en sociedad. Si entendemos por corrupción el uso de un cargo en beneficio propio y en desmedro del bien común (Rose-Ackerman y Palifka, 2016), debemos reconocer que algunas prácticas como el maltrato laboral, la discriminación, o el uso o mal uso de información, no caben dentro de esas categorías. Al asumir la perspectiva de la integridad, ese tipo de prácticas también son vistas como conductas impropias y respecto de las cuales hay que actuar, ya sea tratando de evitarlas o sancionándolas. Huberts y van Montfort (2020a: 453) entregan un listado de prácticas que contravienen la integridad, tales como recibir sobornos, los favoritismos (tales como nepotismos o patronazgo), fraude y robo de recursos, conflictos de intereses, tanto producto de la recepción de regalos como actividades paralelas, el uso impropio de la autoridad, el uso y mal uso de información, dilapidar recursos públicos, trato indecente de las o los colegas, y el comportamiento inadecuado en la vida privada. Como vemos, este listado 
combina prácticas de corrupción evidentes con otras que no necesariamente lo son, pero que evidencian un mal comportamiento ético de las o los funcionarios. A esta enumeración podríamos sumar el extenso listado de prácticas de "maladministración" (Caiden, 1991), donde se pueden evidenciar acciones, dentro de las organizaciones públicas, que expresan falta de integridad por parte de los involucrados.

La instalación de sistemas de integridad viene a enfrentar el tema de la lucha contra la corrupción desde una perspectiva diferente a la meramente legal. En la literatura, al primer enfoque se le denomina de la "integridad (integrity)" o high road (Rohr, 1989). Algunos autores hablan de este enfoque también como de "valores", toda vez que busca estimular la reflexión valórica para estimular conductas íntegras (Paine, 1994). Por el contrario, la estrategia tendiente a relevar el componente legal o normativo se llama de la "obedencia (compliance)" o low road. Nos parece que en el caso particular chileno, la instalación de los sistemas de integridad, partiendo con los códigos de ética, responde más a la lógica de la integridad, puesto que, como señala Maesschalck (2004), esta estrategia se centra en los controles internos de las y los funcionarios apoyando el proceso de reflexión moral ante situaciones de dilemas éticos. El tipo de código de ética elaborado e implementado en Chile de manera participativa incorpora un enfoque práctico orientado a la reflexión valórica, y se relaciona con otros elementos de gestión de la ética como el involucramiento de los miembros de la organización, capacitaciones sobre dilemas éticos, talleres de socialización sobre la relevancia de la integridad, etc. El sistema de integridad organizacional como un todo combina elementos de integridad con los de obediencia, siendo consistentes con las tendencias globales sobre la materia (Maesschalck y Bertók, 2009).

Respecto de la arquitectura de los sistemas de integridad, se puede señalar que son tres los modos más conocidos de diseño de estas formas de intervención: los sistemas nacionales de integridad (national integrity systems), los sistemas locales de integridad (local integrity systems) y los sistemas a nivel de organizaciones públicas. En el primer caso, fue Pope (1996) quien en un trabajo para Transparencia Internacional presentó y desarrolló el concepto. Este tipo de sistemas de integridad se plantean como mecanismos comprehensivos de intervenciones y controles a nivel país para lograr el control de la corrupción y el reforzamiento ético de las y los funcionarios. Ejemplos de estos sistemas de integridad son los del Reino Unido (Home Office, 2018) y Finlandia (Anti-Corruption.fi, 2021) en Europa, y en América Latina el Sistema Anticorrupción de México (sNA, 2021) y el de Brasil (ENCCLA, 2021). El segundo tipo de diseño de sistemas de integridad 
es el de los sistemas a nivel local o sistemas locales de integridad. En este caso, se diseñan los mismos componentes de todo sistema de integridad, pero el ámbito de acción es el local (Huberts et al., 2008; Macaulay et al., 2014). Finalmente, existen los sistemas de integridad de las organizaciones públicas, que buscan instalar, a través de una estrategia sistémica, componentes que aseguren un marco de integridad para las instituciones públicas (Maesschalck y Bertók, 2009). En cada país podrán convivir estos tipos de sistemas, con mayor o menor nivel de coordinación o formalidad.

Otro elemento relevante del diseño de los sistemas de integridad guarda relación con el objeto de control. A este respecto, los sistemas de integridad ofrecen dos tipos de sujetos de control dominantes. Por un lado, existen sistemas de integridad que buscan controlar a la clase política, en particular a las autoridades electas políticamente, mientras existen otros sistemas que se preocupan del control de las y los funcionarios públicos o del sistema local en general. En el análisis de Quesada et al. (2013) se muestra en particular cómo se aplicó el sistema local de integridad en las ciudades españolas, donde, a pesar de estar en funcionamiento, se permitió que ocurrieran importantes casos de corrupción. Como fue bien documentado en un estudio comparado de siete ciudades (Huberts et al., 2008), existen elementos culturales y particulares que llevan al diseño de distintos tipos de sistemas locales de integridad, lo que se expresa en modelos más centrados en agencias autónomas o en sendos códigos de conducta, mostrando resultados disímiles.

\section{Sistemas de integridad organizacionales}

En general, las formas en que se implementan los sistemas de integridad difieren mucho entre los países, particularmente por las orientaciones e intenciones que hacen necesario este tipo de intervención. Si bien la literatura no es muy abundante en relación con la forma de implementar este tipo de sistemas, ya sea topdown o bottom-up, con apoyo de agencias internacionales o no, o alguna otra forma, existen importantes trabajos respecto a los elementos que debe tener esa implementación para que los sistemas de integridad cumplan su efecto.

En el caso de los sistemas de integridad organizacionales, existen cuatro elementos que deben tomarse en cuenta para que la implementación del sistema tenga éxito. Como se presenta en Maesschalck y Bertók (2009: 27), un sistema de integridad en las organizaciones debe cumplir las siguientes funciones: determinar y definir lo que se entiende por integridad, guiar hacia la integridad, monitorear la integridad y, finalmente, aplicar sanciones en caso de que el sistema no 
CUADRO 1. Funciones de los sistemas de integridad organizacional y sus respectivos instrumentos o componentes

\begin{tabular}{|c|c|c|c|c|}
\hline Funciones & $\begin{array}{c}\text { Determinar y definir } \\
\text { lo que se entiende } \\
\text { por integridad }\end{array}$ & $\begin{array}{c}\text { Guiar hacia la } \\
\text { integridad }\end{array}$ & $\begin{array}{c}\text { Monitorear la } \\
\text { integridad }\end{array}$ & $\begin{array}{c}\text { Aplicar sanciones } \\
\text { en caso de que el } \\
\text { sistema no se cumpla }\end{array}$ \\
\hline $\begin{array}{l}\text { Instrumentos o } \\
\text { componentes } \\
\text { del sistema } \\
\text { de integridad } \\
\text { organizacional* }^{*}\end{array}$ & $\begin{array}{l}\text { Análisis de } \\
\text { riesgos, código de } \\
\text { conducta, política } \\
\text { de conflictos de } \\
\text { interés, política de } \\
\text { regalos, arreglos } \\
\text { postempleo, } \\
\text { medidas } \\
\text { estructurales } \\
\text { (rotación de } \\
\text { funciones), } \\
\text { análisis de dilemas } \\
\text { éticos, consultas } \\
\text { al personalya } \\
\text { interesados, código } \\
\text { de ética, definir } \\
\text { estándares no } \\
\text { escritos sobre la } \\
\text { materia }\end{array}$ & $\begin{array}{l}\text { Capacitaciones } \\
\text { sobre reglas ligadas } \\
\text { a la integridad, } \\
\text { juramento o firma de } \\
\text { una declaración de } \\
\text { integridad, consejo } \\
\text { y asesoría, } \\
\text { capacitaciones sobre } \\
\text { el factor de valores de } \\
\text { la integridad, incluir } \\
\text { la integridad en el } \\
\text { discurso regular, } \\
\text { conducta ejemplar } \\
\text { de los directivos, } \\
\text { coaching y consejo } \\
\text { sobre integridad }\end{array}$ & $\begin{array}{l}\text { Políticas de } \\
\text { denunciantes, } \\
\text { políticas de denuncias } \\
\text { o quejas, inspecciones, } \\
\text { testeos de integridad, } \\
\text { sistemas de alarmas } \\
\text { tempranas, registro } \\
\text { sistemático de quejas, } \\
\text { investigaciones, etc., } \\
\text { encuestas donde se } \\
\text { midan las violaciones } \\
\text { a la integridad y clima } \\
\text { organizacional, } \\
\text { encuestas sobre } \\
\text { dilemas de integridad, } \\
\text { pruebas informales } \\
\text { para verificar dilemas } \\
\text { éticos }\end{array}$ & $\begin{array}{l}\text { Sanciones formales, } \\
\text { procedimientos para } \\
\text { manejar violaciones } \\
\text { a la integridad, } \\
\text { sanciones informales }\end{array}$ \\
\hline
\end{tabular}

Fuente: Adaptado de Maesschalck y Bertók (2009: 28). *En el cuadro original, los autores diferencian los instrumentos o componentes de obediencia y de valores, pero para fines de claridad se presentan ambos en una sola categoría.

se cumpla. Estas cuatro dimensiones requieren un grupo de instrumentos, normas y acciones, orientados a que las instituciones actúen con integridad. El cuadro 1 muestra los instrumentos y componentes para poder implementar un sistema de integridad en una organización de manera eficiente, cumpliendo cada una de las funciones que estos deben abordar.

La correcta implementación de un sistema de integridad organizacional se materializará en la medida en que las cuatro funciones señaladas, y presentadas en el cuadro 1, se cumplan. Lo sistemático de estos esfuerzos organizacionales reside en que se requieren muchos elementos para que el desafío de reforzar la integridad de las y los funcionarios públicos sea alcanzable. En la siguiente sección del artículo presentaremos cómo se implementaron los sistemas de integridad en la administración pública chilena, para luego analizar el grado de implementación de los mismos. 


\section{CONTEXTO}

Si bien el concepto "sistema de integridad" es un término nuevo dentro de la institucionalidad, durante las últimas décadas las organizaciones del sector público chileno han contado con una serie de elementos orientados al fortalecimiento del comportamiento probo por parte del personal de la Administración. Es así como instrumentos jurídicos como el Estatuto Administrativo, la Ley de Acceso a la Información y obligaciones ligadas a las tendencias del compliance a cargo de la Unidad de Análisis Financiero, las capacitaciones sobre probidad o los mecanismos de rendición de cuentas por parte de autoridades y funcionarios se presentaban en la práctica como instrumentos para prevenir la corrupción. Sin embargo, estos elementos se desplegaban, muchas veces, de manera desarticulada y sin una mirada integral para fortalecer el clima ético al interior de las organizaciones.

De manera paralela, una rápida revisión a las medidas anticorrupción implementadas durante las últimas décadas en el sector público permite identificar cómo estas tuvieron un énfasis centrado en la obediencia por sobre la integridad (Lagos y Pliscoff, 2020). Es así como el año 2016 marca un hito en la forma en que el país enfrenta la corrupción. Por primera vez se modifica la estrategia dominante de acción en materia de corrupción, centrada en un enfoque legalista y normativo por uno de integridad que busca un cambio conductual por parte de los miembros de las organizaciones.

El cambio de estrategia en materia de probidad tuvo como antesala el descubrimiento de una serie de escándalos de corrupción vinculados a situaciones de financiamiento irregular a la política. Estos casos dieron a conocer cómo partidos políticos de diversos sectores recibían de manera indirecta recursos de empresas privadas para solventar gastos electorales. Así, se cuestionó la relación entre negocios y política, y se evidenció la necesidad de buscar nuevos caminos para fortalecer la integridad pública (Arís et al., 2020; Consejo Asesor Presidencial contra los Conflictos de Interés, 2015; Contraloría General de la República de Chile, 2020). Si bien Chile no es señalado como un país con corrupción sistémica como otros países de la región (Petersen Cortés, 2020), estos casos tuvieron una gran relevancia social que llevó a modificar prácticas en este ámbito.

En este contexto, la Presidencia de la República da origen a la Comisión Asesora Presidencial contra los Conflictos de Intereses y el Tráfico de Influencias, plataforma desde donde se impulsa la creación de códigos de ética que orienten el comportamiento íntegro del personal en cada una de las entidades públicas y ministerios de la Administración central del Estado. Esa iniciativa fue liderada por la DNSC con el apoyo del Programa de Naciones Unidas para el Desarrollo 
(PNUD) que brindó el apoyo metodológico a un proceso basado en cuatro fases y con un marcado énfasis participativo (Carrizo y Silva, 2017). Este esfuerzo permitió que 256 instituciones contaran con sus primeros códigos de ética elaborados de manera participativa y coordinada con autoridades, personal y las asociaciones de funcionarios y funcionarias.

El desafío de implementar y dar sostenibilidad a los códigos de ética se abordó a partir de 2017 a través de la instalación en cada una de las entidades públicas de un sistema de integridad conformado por cinco elementos claves:

1. Jefe/a superior de la entidad encargado/a de liderar con el ejemplo la implementación del código de ética.

2. Asesores/as técnicos/as encargados/as de entregar orientación experta al jefe/a superior de la institución para la toma de decisiones.

3. Coordinador/a de integridad responsable de crear o implementar acciones y herramientas para fortalecer una cultura ética al interior de la organización.

4. Comité de Integridad con un carácter propositivo y consultivo conformado por representantes de distintas áreas de la organización.

5. Plataforma tecnológica interna y externa donde se realice la socialización de las actividades del sistema y se reciban consultas y denuncias.

Para apoyar la implementación de estos elementos, la DNSC desarrolló capacitaciones para las entidades públicas y emitió cuatro oficios con orientaciones técnicas y operativas para apoyar y dar seguimiento a la correcta implementación de estas recomendaciones (véase el Anexo 1).

\section{METODOLOGÍA}

La presente investigación es de carácter exploratorio-descriptivo y consideró herramientas metodológicas cuantitativas de nivel descriptivo para reconocer la situación actual de las entidades públicas de la muestra seleccionada. Asimismo, se utilizaron herramientas cualitativas para profundizar en la comprensión de la información levantada e identificar factores de éxito y áreas de mejora del proceso.

En una primera etapa se levantó información sobre el grado en que las entidades públicas cumplen el Oficio Ordinario núm. 1316 de 2017 en que se informan acciones vinculadas a la implementación del Sistema de Integridad en cada Institución Pública y los posteriores Oficios Ordinarios núm. 335 de 2018 y núm. 613 de 2018 en que se solicitaban antecedentes vinculados a la implementación de estos sistemas. La información fue requerida a través de solicitudes de acceso a la 
información, lo que asegura que la respuesta recibida contenga los datos oficiales de la institución y sea entregada por el equipo o persona funcionaria designada para tal función. En específico, la solicitud consistió en un cuestionario de trece preguntas estandarizadas para todas las instituciones (véase el Anexo 2).

Si bien la DNSC informó sobre la creación de 256 códigos de ética (DNSC, 2017), esta investigación considerará como universo de estudio las 196 instituciones cuyos códigos de ética se encontraban publicados en el sitio de la DNSC el 10 de diciembre de 2019. Esto debido a que no se tuvo acceso al listado detallado de las 256 instituciones a las cuales los comunicados institucionales hicieron mención.

A partir de los datos obtenidos y para dar paso a la segunda etapa de metodologías cualitativas, se sistematizaron clusters de instituciones con el objetivo de caracterizar los distintos grados de implementación de los sistemas de integridad. Como resultado de este ejercicio, se identificaron cuatro grupos de instituciones que reflejan cuatro niveles de implementación de los mecanismos anticorrupción.

Para profundizar en la información generada en la primera etapa y con los objetivos de identificar buenas prácticas y conocer en detalle el grado de implementación de aquellas entidades más avanzadas, se seleccionaron 11 instituciones pertenecientes a los niveles más avanzados de implementación a través de un muestreo por conveniencia y se les invitó a participar en una entrevista a cargo del equipo de investigación. Se obtuvo respuesta de ocho instituciones a las cuales se les aplicó un cuestionario semiestructurado que tenía como ejes el diagnóstico de la realidad institucional, la identificación de factores de éxito y el reconocimiento de dificultades en la implementación de los elementos del sistema de integridad. El detalle de estas actividades se puede revisar en el Anexo 4.

La información recopilada en el marco de esta investigación ha sido evaluada con base en dos parámetros. El primero fue el cotejo con aquellos lineamientos establecidos por la DNSC a través de sus oficios a entidades públicas, a fin de determinar si "en la forma" se cumplieron los lineamientos para lo que se utilizaron estadísticos descriptivos clásicos. El segundo serán aquellas buenas prácticas promovidas por teóricos de la gestión de la ética y de las organizaciones de la comunidad internacional y que serán analizadas principalmente a partir de la revisión documental mencionada anteriormente.

\section{RESULTADOS GENERALES}

La primera etapa de la investigación contempló el envío de una solicitud de acceso a la información a 196 instituciones que conformaron el universo de estudio. Se recibió respuesta de 193 instituciones, las que conformarán la muestra de esta 
investigación. Los resultados señalados a continuación se presentarán en dos apartados: infraestructura del sistema y funcionamiento operativo.

\section{Instalación de la infraestructura del sistema del sistema de integridad}

La estructura formal sugerida por la DNSC contempla una infraestructura conformada por los siguientes elementos:

- Comité de Integridad conformado por funcionarios/as representantes de la institución que ejerce como un órgano de carácter propositivo y consultivo.

- Asesores/as técnicos/as encargados/as de entregar orientación técnica al jefe/a superior de la institución para la toma de decisiones.

- Coordinador/a de integridad responsable de crear e implementar acciones y herramientas para fortalecer una cultura ética al interior de la organización.

- Plataforma tecnológica interna con el registro de las consultas y denuncias realizadas.

- Plataforma tecnológica externa donde se publique el código de ética, una sección de preguntas frecuentes y noticias de actividades realizadas en el marco del sistema.

- Canal de consultas y denuncias que reciba aquellas inquietudes, dilemas e inobservancias éticas alertadas por los miembros de la organización.

En el cuadro 2 se señalan los criterios de evaluación utilizados para cada elemento y los resultados obtenidos.

CUADRO 2. Resultados de la implementación de elementos del sistema de integridad

\begin{tabular}{lccc}
\hline \multicolumn{1}{c}{ Criterio de evaluación } & Si (\%) & No (\%) & N/R (\%) \\
\hline Comité de integridad aprobado a través de resolución & 92 & 6 & 2 \\
Asesores/as técnicos/as designados por resolución & 86 & 10 & 4 \\
Designación de coordinador de integridad por resolución & 91 & 7 & 3 \\
Plataforma tecnológica externa con código de ética & 85 & 11 & 4 \\
Plataforma tecnológica interna con sección del sistema & 76 & 17 & 7 \\
Canal de consultas y denuncias & 77 & 16 & 7 \\
Canal de consultas y denuncias anónimo & 54 & 39 & 7 \\
Canal de consultas y denuncias abierto a solicitudes externas & 47 & 47 & 6 \\
\hline
\end{tabular}

Fuente: Elaboración propia. 
En general se evidencia un alto grado de implementación de la infraestructura de un sistema de integridad institucional. Así, destaca que 92 por ciento de las instituciones analizadas cuenta con un comité de integridad, 91 por ciento de las instituciones ha designado a una persona en el rol de coordinador/a de integridad y 86 por ciento de las instituciones tienen designados a profesionales como asesores/as técnicos/as. Un grado menor de implementación poseen los elementos asociados a las plataformas tecnológicas y los canales de consultas y denuncias anteriormente mencionados. En específico, la plataforma externa se encuentra desarrollada en 85 por ciento de las instituciones, mientras que la plataforma interna se encuentra implementada en 76 por ciento de las organizaciones estudiadas. Finalmente, los canales de consultas y denuncias se encuentran disponibles en 77 por ciento de las entidades analizadas.

Tres aspectos complementarios al mero cumplimiento de estos elementos, llaman la atención. En primer lugar, 75 por ciento de las instituciones crearon su comité de integridad en el año 2018 luego del primer oficio de la DNSC en que se solicitaba emanar un informe de la implementación de estos elementos. En segundo lugar, la gran mayoría de las instituciones cuenta con un canal en que es necesario identificarse para ingresar una solicitud y solo 39 por ciento recibe solicitudes anónimas. En tercer lugar, las instituciones se distribuyen en igual proporción entre aquellas que aceptan solicitudes de personas externas a la organización de aquellas que no lo permiten.

A partir de esto, en el siguiente apartado se presentan los resultados del estudio del grado de funcionamiento operativo de estos elementos.

\section{Funcionamiento operativo de los sistemas de integridad}

El presente estudio - junto con conocer el grado de implementación formal o documental de cada sistema analizado en el punto anterior-, tiene por objetivo entender el grado de funcionamiento práctico que cada sistema tiene dentro de las organizaciones. Para esto, se seleccionaron dos elementos a los cuales es posible asociar un indicador que refleja la cantidad de actividad o movimiento que el sistema puede tener al interior de la entidad. A continuación, se señalan los dos elementos seleccionados con sus respectivos indicadores (véanse las gráficas 1,2 y 3 ).

Estos indicadores permiten complementar la información levantada en el primer punto acerca del cumplimiento formal o documental del sistema y tener evidencia para analizar cómo están funcionando en la operación diaria estos instrumentos de gestión ética. 


\section{a) Comité de integridad}

GRÁFICA 1. Número de reuniones que el comité ha tenido desde su creación hasta el 10 de diciembre de 2019

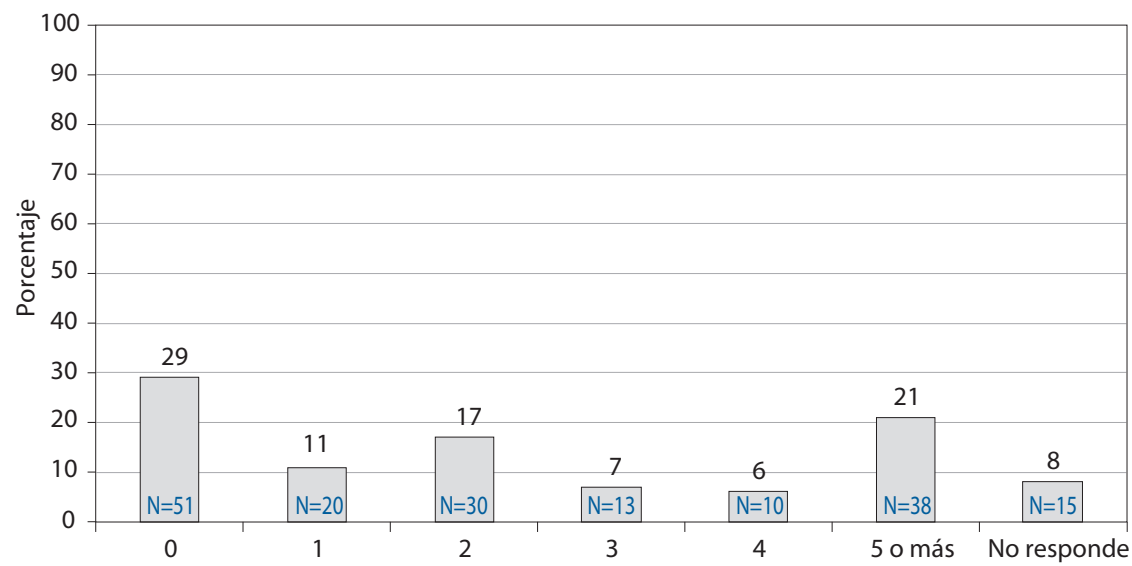

Fuente: Elaboración propia.

\section{b) Canal de consultas y denuncias}

GRÁFICA 2. Número de consultas que han recibido aquellas instituciones con canal de consultas y denuncias

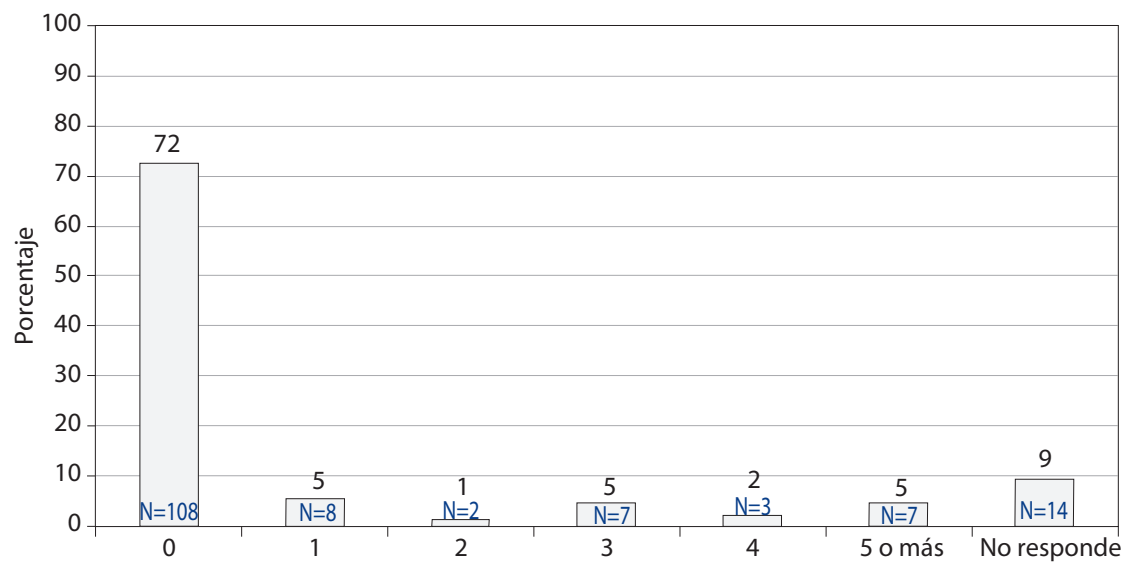

Fuente: Elaboración propia. 
GRÁFICA 3. Número de denuncias que han recibido aquellas instituciones con canal de consultas y denuncias

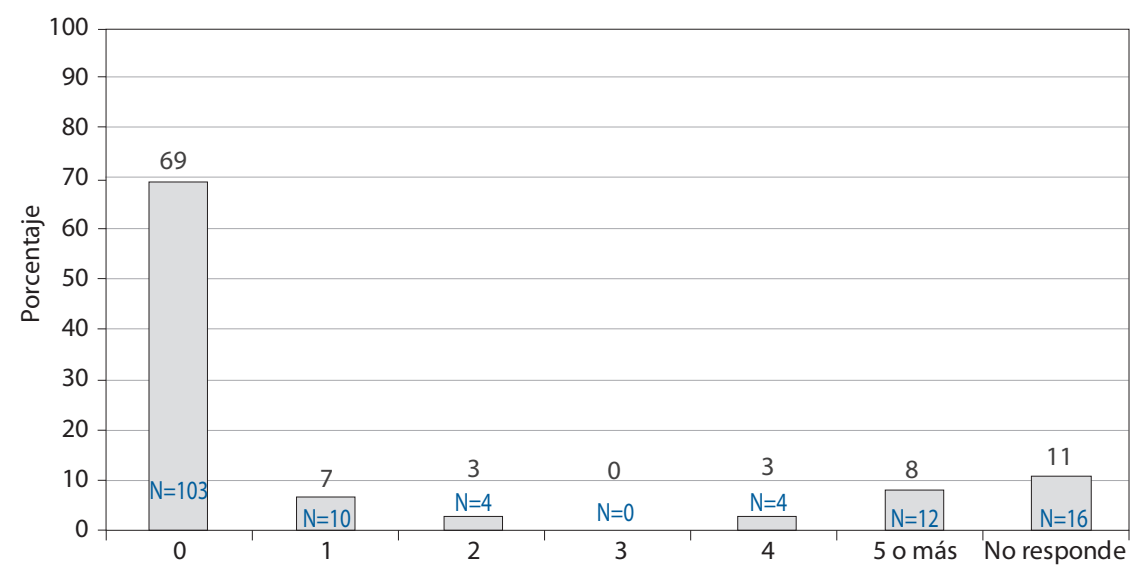

Fuente: Elaboración propia.

Los resultados evidencian que los comités de integridad de la muestra han sesionado en promedio tres veces en el periodo analizado, con una desviación estándar de 3.58 y una moda de 0 veces. Por otro lado, los canales de consultas han recibido en promedio 3.3 consultas con una desviación estándar de 19 y una moda de 0. Finalmente, los canales de denuncia han recibido un promedio de $1.6 \mathrm{de}-$ nuncias con una desviación estándar de 5.4 y una moda de 0 . Estos estadísticos evidencian la diversidad existente en el funcionamiento operativo de los sistemas a nivel nacional, por lo que en la siguiente sección se analizarán en detalle los datos obtenidos proponiendo una clasificación de las entidades analizadas.

A partir de estos resultados, en los siguientes apartados se examinará en detalle la situación diagnosticada, lo que permitirá, finalmente concluir cómo esta situación particular puede ser un aporte en la discusión sobre la prevención de la corrupción y el fortalecimiento de la integridad organizacional.

\section{ANÁLISIS DEL NIVEL DE IMPLEMENTACIÓN}

A partir de los indicadores señalados en el punto anterior y considerando como base las variables asociadas al número de reuniones y la suma de consultas y denuncias recibidas por cada institución, se realizó un análisis de cluster para identificar distintos niveles de implementación de los sistemas. 
GRÁFICA 4. Niveles de implementación de los sistemas de integridad

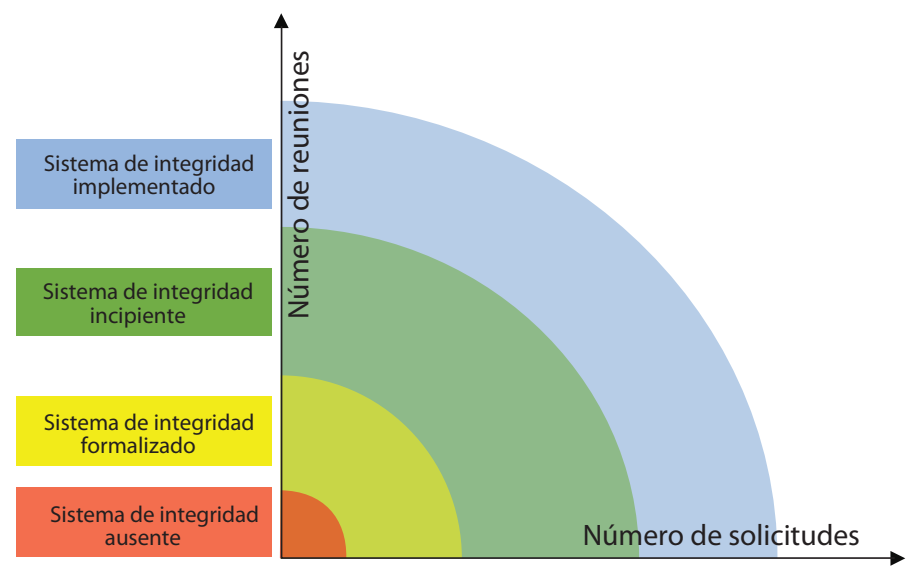

Fuente: Elaboración propia.

Luego de considerar 31 instituciones como casos perdidos, ya que no se poseía información de las dos variables, el análisis permitió identificar cuatro niveles de implementación. Cabe señalar que no fue posible establecer una relación asociada algún tipo de sector o tamaño institucional y el nivel de implementación; por el contrario, las instituciones se distribuyen, en general, de manera equitativa del sector que vienen y el grado de implementación de los elementos del sistema.

La gráfica 4 ilustra los cuatro niveles de implementación identificados luego del análisis de cluster. El detalle de la conformación de los cuatro cluster puede ser revisado en el Anexo.

Nivel A: Sistema de Integridad Ausente (25 instituciones)

Las instituciones que se encuentran en este nivel corresponden a 15 por ciento de la muestra analizada y se caracterizan por no poseer al menos uno de los elementos base de la infraestructura del sistema definido por la DNSC. Es decir, son instituciones que, si bien cuentan con un código de ética elaborado participativamente, no cuentan con un comité de integridad o un canal de recepción de consultas y denuncias. Tal como se explicó en las primeras secciones de este trabajo, estas herramientas son indispensables para darle sustento, coherencia y sostenibilidad al código de ética, por lo que no podría establecerse que estas instituciones cuenten con un sistema de integridad institucional. 
Resulta evidente, entonces, que para transitar a un estadio donde se pueda afirmar la existencia de un sistema es indispensable contar con un comité y un canal de consultas y denuncias que formalicen una plataforma organizacional sobre la que se implemente el código de ética. Esta premisa fue reafirmada en las entrevistas realizadas a las y los coordinadores de integridad, donde se destacó que la existencia del Comité "ha mantenido vivo el sistema", ya que la autoridad reconoce a esta instancia como un actor relevante dentro de la institución. Así, le ha asignado tareas específicas vinculadas al fortalecimiento de la ética organizacional.

Nivel B: Sistema de integridad formalizado (93 instituciones)

En este nivel se encuentra 57 por ciento de las instituciones analizadas, las que se caracterizan con dar cumplimiento a los criterios establecidos por la DNSC sobre la infraestructura del sistema; es decir, cuentan con un comité de integridad formalizado a través de una resolución y un canal de consultas y denuncias al que se puede acceder por la plataforma tecnológica interna. Sin embargo, estas instituciones presentan un bajo nivel de actividad de ambos elementos. Es decir, si bien se encuentran formalizados, el funcionamiento operativo de los mismos es escaso, con un número muy bajo de sesiones del comité de integridad y un canal de consultas y denuncias que ha recibido desde su creación escasas solicitudes. Esto impide establecer que el sistema esté funcionando de manera permanente, coherente y con un impacto en el clima ético organizacional.

Este asunto fue señalado por los coordinadores de integridad entrevistados, en términos de reconocer que la articulación de un canal de denuncias electrónico anónimo ha aumentado la recepción de solicitudes. Esta situación a su vez ha motivado a los comités de integridad a desarrollar nuevas acciones que fortalezcan el funcionamiento del sistema.

A partir de esto, las instituciones que se encuentran en este nivel de implementación debiesen disponer las gestiones necesarias para reactivar el funcionamiento de su comité de integridad como una instancia continua en el quehacer institucional. $\mathrm{Al}$ mismo tiempo debiesen aumentar las acciones de capacitación y socialización de sus canales de consultas y denuncias como una manera de promover que los miembros de la organización los tengan presentes en el trabajo diario y recurran a ellos cuando se enfrenten a un dilema ético.

Nivel C: Sistema de integridad incipiente (39 instituciones)

Este nivel de implementación está conformado por 23 por ciento de las instituciones analizadas que pueden demostrar el desarrollo documental de los elemen- 
tos base para un sistema de integridad y evidenciar el funcionamiento mínimo de estos elementos al interior de la organización. En este sentido, las tasas asociadas al funcionamiento regular y permanente del comité de integridad en el periodo analizado no permiten asegurar que esta instancia se desenvuelva de manera regular y constante en el quehacer institucional. Mientras que el número de consultas y denuncias recibidas evidencia que es una herramienta con escaso uso por parte de los miembros de la organización.

De este modo, las entidades que se encuentran en este nivel tienen el desafío de transitar hacia un nivel de mayor implementación que permita asegurar que el sistema de integridad institucional funcione de manera sostenible y con impacto en la organización. Esto es posible mediante dos vías. En primer lugar, asegurar la consolidación del comité de integridad como una instancia relevante al interior del sistema, fortaleciendo su trabajo para aumentar el impacto de su rol en el robustecimiento del funcionamiento operativo del sistema. Por otro lado, estas organizaciones enfrentan el desafío de identificar y comprender aquellos factores que imposibilitan un uso intensivo de los canales de consultas y denuncias por parte de los miembros de la organización. Esto es indispensable para avanzar de un sistema de integridad genérico a un sistema ad-hoc a las necesidades y características de la organización.

Este último punto ha sido resaltado por las y los coordinadores de integridad entrevistados, quienes destacan la importancia de que los funcionarios y funcionarias confíen en el funcionamiento del sistema, especialmente en que el canal de consultas y denuncias asegure la reserva de identidad de quien ingresa una solicitud. En este mismo sentido, se reconoce que uno de los problemas para la consolidación del sistema se vinculaba al grado de desconfianza que los funcionarios tenían de que su denuncia se tradujese en sanciones concretas a los denunciados.

Nivel D: Sistema de integridad implementado (7 instituciones)

En el último nivel de implementación se encuentra 4 por ciento de las entidades analizadas. En esta situación se encuentran instituciones que cuentan con los elementos de un sistema de integridad formalizados y pueden evidenciar el funcionamiento operativo de los mismos a través de un registro de actividades permanentes tanto en el comité como los canales de consulta y denuncia. En este sentido, las organizaciones que se encuentran en este estadio se caracterizan por contar con un comité que se reunió de manera periódica y permanente durante el periodo de estudio junto con un canal de consultas y denuncias que recibió un número de solicitudes que evidencian un funcionamiento operativo regular del mismo. 
Tal como se señaló en el inicio de esta sección, no ha sido posible observar una distribución especial que vincule a algún sector en particular con algún nivel específico de implementación. Por el contrario, en este nivel es posible encontrar instituciones provenientes de los ministerios de Hacienda (tres instituciones), Salud (dos instituciones), Economía (una institución) y Deportes (una institución). De igual forma, las instituciones poseen tamaños muy disímiles entre 200 (Chile Compra) y más de 5 mil funcionarios (Servicio de Impuestos Internos), por lo que no es posible establecer una vinculación entre el tamaño de la institución y el nivel de implementación en que se ubican.

Luego de identificados estos cuatro niveles de implementación, es importante analizar algunos factores de contexto o componentes organizacionales que las personas entrevistadas señalaron como relevantes y que permiten comprender las razones por las que las instituciones han logrado un grado más alto de implementación del sistema frente a otras entidades que han tenido mayores dificultades en la misma misión.

Un primer elemento es la presencia de factores de anclaje del sistema a la organización. A partir de las entrevistas realizadas se pudo identificar la existencia de dos elementos principales que permiten afianzar de mejor manera los elementos del sistema de integridad al quehacer institucional. El primero de ellos hace referencia al perfil y las características especiales de quien desempeña el rol de coordinador/a de integridad. Habilidades como el liderazgo, el compromiso activo y la comunicación efectiva con los distintos miembros de la institución parecen ser factores con un alto impacto que facilitan la implementación exitosa del sistema en la organización. El segundo factor de anclaje es la vinculación del sistema con una temática estratégica para la institución como lo puede ser la existencia anterior de mecanismos de compliance o comités vinculados a temáticas de género o no discriminación ya consolidados al interior de la organización. En este sentido, las experiencias exitosas identificadas parecían tener una fuerte vinculación con otros mecanismos institucionales previos que al gestionarlos de manera integral adquirían mayor fuerza e impacto en la organización.

Lo anterior se ejemplifica al identificar que las personas entrevistadas no realizaban una distinción clara o evidente entre el sistema de integridad promovido por la DNSC e iniciativas vinculadas, pero con fines distintos como el sistema antifraude promovido por la Unidad de Análisis Financiero o los comités de no discriminación.

Otro elemento distintivo identificado en las entrevistas de aquellas organizaciones con prácticas exitosas de implementación guarda relación con el rol de la 
jefatura superior de servicio y la instrumentación que realiza del comité de integridad. En términos concretos, se pudo reconocer que aquellos comités de integridad con una alta actividad trataban en sus reuniones temáticas vinculadas a la ética, pero priorizadas y asignadas como tareas especiales al comité. Por ejemplo, uno de los entrevistados mencionó explícitamente cómo la creación de un protocolo de no discriminación o una actualización de las políticas de transparencia institucional asignadas al comité de integridad fueron tareas que dieron sostenibilidad en el tiempo a esta instancia y les permitieron asumir nuevas tareas para mantener activa su existencia. Si bien estas situaciones contradicen las recomendaciones entregadas por la DNSC tendientes a no mezclar las temáticas de integridad con otras materias de gestión institucional, la evidencia recogida en las entrevistas indica que utilizar el comité de integridad como plataforma coordinada de estas distintas iniciativas puede tener buenos resultados para la óptima implementación del sistema.

En último lugar, a través de las entrevistas se identificó el rol que la DNSC ha tenido en la implementación exitosa de los sistemas de integridad. A partir de las entrevistas se pudo reconocer una percepción altamente positiva sobre el papel desempeñado por esta institución en el proceso de elaboración del código de ética, percepción que se ha ido diluyendo con el paso del tiempo. Esto se traduce en que las instituciones entrevistadas reconocen que la asesoría técnica entregada por la DNSC ocurre ante solicitudes específicas de cada coordinador/a, sin que se reciban lineamientos permanentes y generales sobre pasos a seguir para el perfeccionamiento del sistema. En este sentido, y a partir de lo señalado por todas las personas entrevistadas, parece trascendental reconocer que la implementación exitosa se explica más bien por factores internos a la organización más que por elementos o actores externos a la entidad.

\section{CONCLUSIONES}

Luego del análisis efectuado, con la recopilación de los antecedentes sobre el funcionamiento de estos tres años de los sistemas de integridad en la administración central del Estado chileno, resulta pertinente hacer algunas reflexiones sobre el modo en que Chile decidió para tener estos sistemas. El primer elemento que resulta de la revisión de los datos, y del análisis de las prácticas, es que, si bien se optó por un sistema de integridad organizacional, vale decir que en los sistemas de integridad para cada una de las agencias o servicios públicos y sus ministerios, ajustados a las particularidades de cada servicio, se definieron lineamientos desde las autoridades centrales, en particular del Ministerio de Hacienda, para que cada 
servicio y ministerio trabajara en estos sistemas. Es más, el hecho de que el Ministerio de Hacienda, a través de su servicio dependiente, la DNSC, asesorado por el PNUD, solicitara a todos los servicios confeccionar los códigos de ética, demuestra la orientación que el gobierno quiso dar. Esto porque si bien se obliga a todos a tener códigos de ética, se implementa una metodología participativa, donde tanto directivos como funcionarios y funcionarias trabajan en pos de crear un instrumento pertinente y ajustado a las necesidades de la organización. El haber obligado a hacer los códigos de otra manera hubiese ido en contra de la idea misma de permitir que la reflexión sobre integridad se produzca en cada espacio particular. Desde un punto de vista de las funciones de los sistemas de integridad organizacionales propuestas por Maesschalck y Bértok (2009), este proceso permite que cada servicio y ministerio pueda definir y reflexionar sobre lo que ellos entienden por integridad.

El segundo elemento destacable del modelo chileno de implementación de sistemas de integridad es que ha estimulado la creación de canales de consultas y denuncias, que vayan más allá de los tradicionales canales formales propios de las entidades públicas. De acuerdo con las recomendaciones generales de las entidades internacionales como la OCDE (2020) o el Consejo de la Unión Europea (UE, 2019), es fundamental que existan canales particulares para que se reciban consultas y denuncias en materia de integridad, tanto para funcionarios como para la ciudadanía. Como pudimos ver en las cifras, si bien un número significativo de los servicios tiene canales, no en todos existe un uso de estos. Sin embargo, la sola existencia de estos canales, como pudo verificarse con los servicios de mayor desarrollo de sus sistemas, da dinamismo a los sistemas de integridad, puesto que generan datos e información para que los comités de integridad trabajen, ya sea analizando y canalizando las denuncias como conociendo materias que eventualmente pudiesen ser tomadas en cuenta para el mejor funcionamiento del sistema.

Un tercer elemento que surge como conclusión del trabajo desarrollado en este documento es el rol de la DNSC como ente encargado del monitoreo del sistema. La implementación de los sistemas de integridad es una combinación de elementos comunes, con aspectos particulares. Así como no debería existir un código de ética para todos los servicios públicos, por las particularidades que cada servicio debe enfrentar, tampoco deben existir normas diferentes acerca de discriminación, acoso u otro tipo de actos que afectan la dignidad de cualquier funcionario, independientemente del servicio o ministerio donde se cumplen las labores. Una entidad central, como la DNsC, puede apoyar a establecer los linea- 
mientos generales y específicos que permitan patrones comunes entre los sistemas, pero que también den cabida a las especificadas. Del mismo modo, la existencia de un servicio encargado del monitoreo del proceso, más allá del papel que en los hechos ha desempeñado, ayuda a verificar que las cuatro funciones ya comentadas de los sistemas de integridad organizacionales se cumplan.

$\mathrm{Al}$ mismo tiempo, junto con esos elementos positivos que se pueden destacar, se reconocen varias oportunidades de mejora que la presente investigación permitió evidenciar, tales como que la priorización política, organizacional y técnica demostrada al inicio del proceso de implementación de estos mecanismos anticorrupción se ha ido diluyendo con el paso del tiempo. En la actualidad es necesario robustecer el liderazgo estratégico y el compromiso ante la integridad en dos niveles. En primer lugar, resulta indispensable promover la responsabilidad organizacional para perfeccionar y consolidar la implementación de cada sistema de integridad institucional. Sin este compromiso de nivel estratégico resultará imposible continuar con el exitoso proceso iniciado en la elaboración de los códigos de ética. En segundo lugar, la información recopilada sobre la experiencia organizacional de los últimos años evidencia la necesidad de perfeccionar el liderazgo técnico de la DNSC como organismo rector de materias de integridad en las instituciones del gobierno central. La ausencia de este rol de asesoría técnica ha desincentivado y limitado la consolidación de los mecanismos anticorrupción en las entidades estudiadas.

En la misma línea, las distinciones teóricas propuestas por Maesschalck y Bertók (2009) en las primeras páginas de este documento permiten establecer un marco de análisis en el que se identifican fortalezas y aspectos pendientes del proceso para la consolidación de los sistemas de integridad. Es así como el proceso de elaboración de códigos de ética permitió avanzar en la determinación y definición de qué se entiende por integridad de manera específica y aplicada a la realidad y cultura organizacional de cada institución. Esto fue complementado con la instalación a gran escala de los elementos básicos de un sistema de integridad que den sostenibilidad a estas definiciones institucionales. Como resultado en la actualidad cifras cercanas a 90 por ciento de las instituciones analizadas cuenta con comité de integridad, un coordinador/a, una plataforma de socialización y canales de consultas y denuncias.

Sin embargo, aún está pendiente avanzar en las siguientes fases de implementación de estos mecanismos anticorrupción. En primer lugar, orientar y guiar a los miembros de la organización hacia la integridad. En esto resulta indispensable fortalecer las capacitaciones como herramientas, no solo de fortalecimiento del 
clima ético institucional, sino, al mismo tiempo, como un instrumento para darle movimiento al funcionamiento operativo del sistema. Las actividades de formación en materia de anticorrupción pueden ser de amplio alcance, abarcando desde conocimientos jurídicos para enfrentar las malas prácticas administrativas hasta entrenamiento ético que busquen modificar las inobservancias éticas de los miembros de una organización. Todas estas formas de capacitación son reconocidas por múltiples organismos internacionales (OEA, 1996; OCDE, 2020; ONU, 2004) como instancias indispensables para fortalecer la gestión ética organizacional. Sin embargo, en la institucionalidad chilena no existe evidencia de que estén siendo utilizadas de manera óptima como catalizadores y soporte de la consolidación de los sistemas de integridad.

En este mismo sentido, la investigación permitió identificar la necesidad de avanzar en el establecimiento de mecanismos para monitorear la integridad. Además de avanzar en el fortalecimiento de reglas y mecanismos de orientación hacia la integridad, resulta conveniente perfeccionar el funcionamiento de los mecanismos de reportabilidad de las inobservancias éticas. La evidencia obtenida refleja que no existe una estandarización respecto a las características de los canales de consultas y denuncias. En la mayoría de los casos las instituciones no permiten el ingreso de solicitudes anónimas, lo que podría desincentivar el uso de estos mecanismos por parte de quienes conocen actos de faltas a la integridad. De igual forma, existen instituciones que permiten el ingreso de solicitudes de personas externas a la organización, mientras que en otras el canal está restringido a funcionarios y funcionarias de la entidad. La diversidad en estos mecanismos debiese ser analizada en profundidad para evaluar su impacto organizacional e identificar buenas prácticas que permitan estandarizar los canales de consultas y denuncias y dar paso a mecanismos de monitoreo a nivel organizacional pero también por sectores agregados e incluso una consolidación nacional. Todo esto permitiría generar una retroalimentación virtuosa en pos del fortalecimiento y la consolidación de los sistemas de .

A cinco ańos de finalizado el trabajo del Consejo Asesor Presidencial contra los Conflictos de Interés, el Tráfico de Influencias y la Corrupción, este trabajo permitió evaluar la implementación de una de las medidas administrativas que este consejo propuso y que significó un cambio en la manera en la cual la institucionalidad fortalece la integridad pública. El modelo de prevención de la corrupción organizacional a través de sistemas de integridad organizacional ha permitido impulsar progresivamente mecanismos concretos para la gestión ética. El riesgo que detectamos, a propósito de que se hace evidente que el impulso 
inicial no se ha mantenido en el tiempo, es que se pase a una fase en que algunas autoridades, y especialmente muchos funcionarios y funcionarias, sientan que los requerimientos que se les exigen para demostrar el funcionamiento de los sistemas organizacionales de integridad son solo formalidades y no ayudan realmente a reforzar la integridad en las instituciones públicas. Como bien señala Maesschalck (2019: 160), este es uno de los caminos que se toman cuando se instalan sistemas de integridad, en lo que denomina "no tomarse la ética y la gestión en ética seriamente" (p. 160). Cuando se entra en este camino, las acciones de los sistemas de integridad corren el riesgo de rutinizarse y transformarse en un mero "checklist" para mostrar a otros que se está haciendo algo. Es fundamental retomar el liderazgo y alentar a las instituciones que están más avanzadas a seguir en el buen camino e impulsar a las otras en esa dirección. Estas reflexiones, a partir del caso chileno, pueden ser una experiencia valiosa tanto para Chile como para el resto de los países de la región que inician o han iniciado procesos para fortalecer la integridad pública. G气̂

\section{REFERENCIAS}

Andersson, Staffan y Paul M. Heywood (2009), "The Politics of Perception: Use and Abuse of Transparency International's Approach to Measuring Corruption”, Political Studies, 57(4), pp. 746-767.

Andersson, Staffan (2017), "Beyond Unidimensional Measurement of Corruption”, Public Integrity, 19(1), pp. 58-76, Dor: 10.1080/10999922.2016.1200408.

Andersson, Staffan y Frank Anechiarico (2019), Corruption and Corruption Control: Democracy in the Balance, 2a. ed., Nueva York, Taylor \& Francis.

Arellano-Gault, David (2017), "Corrupción como proceso organizacional: Comprendiendo la lógica de la desnormalización de la corrupción”, Contaduría y Administración, 62(3), pp. 810-826.

Anti-Corruption.fi (2021), Combating Corruption in Finland, Helsinki, Ministerio de Justicia, disponible en: https://korruptiontorjunta.fi/en/combating-corruption-in-finland [fecha de consulta: 23 de abril de 2021].

Arís, Manuel, Eduardo Engel y María Jaraquemada (2020), Reformas anticorrupción en Chile 2015-2017: Cómo se hizo para mejorar la democracia, Bogotá y Santiago de Chile, Fundación Konrad Adenauer/Espacio Público, pp. 1-106.

Bautista, Óscar Diego (ed.) (2015), Ética pública frente a corrupción, Toluca de Lerdo, Instituto de Administración Pública del Estado de México.

Caiden, Gerald (1991), "What Really Is Public Maladministration?” Public Administration Review, 51(6), pp. 486-493. 
Carrizo, Daniella y Francisco Silva (2017), Chile en la implementación transversal de códigos de ética pública: Una metodología participativa, Santiado de Chile, Ministerio de Hacienda.

Consejo Asesor Presidencial contra los Conflictos de Interés, el Tráfico de Influencias y la Corrupción (2015), Informe final, Santiago de Chile.

Contraloria General de la República de Chile (2020), Radiografía de la corrupción: Ideas para fortalecer la probidad en Chile, Santiago de Chile, Contraloría General de la República de Chile.

DNSC (Dirección Nacional del Servicio Civil) (2017), "Más de 250 servicios públicos entregan sus Códigos de Ética a la Presidenta Michelle Bachelet”, 20 de junio, Santiago de Chile, Ministerio de Hacienda- DNSC, disponible en: https://www.serviciocivil.cl/ noticias/servicio-civil/mas-de-250-servicios-publicos-entregan-sus-codigos-de-etica-ala-presidenta-michelle-bachelet/ [fecha de consulta: 19 de septiembre de 2020].

ENCCLA (Estratégia Nacional de Combate à Corrupção e à Lavagem de Dinheiro) (2021), Brasilia, MJsp, disponible en: https://www.justica.gov.br/sua-protecao/lavagem-dedinheiro/enccla [fecha de consulta: 23 de abril de 2021].

Gilman, Stuart C. (2005), Ethics Codes and Codes of Conduct as Tools for Promoting an Ethical and Professional Public Service: Comparative Successes and Lessons, Washington, D.C., Banco Mundial.

Heywood, Paul, Heather Marquette, Caryn Peiffer y Nieves Zuñiga (2017), Integrity and Integrity Management in Public Life, University ot Nottingham/University of Birmingham.

Huberts, Leo (2018), "Integrity: What It Is and Why It Is Important", Public Integrity, 20(4), Dor: $10.1080 / 10999922.2018 .1477404$.

Huberts, Leo y Alain Hoekstra (eds.) (2016), Integrity Management in The Public Sector-The Dutch Approach, La Haya, Dutch National Integrity Office.

Huberts, Leo y André van Montfort (2020a), "Building Ethical Organisations: The Importance of Organisational Integrity Systems", en Adam Graycar, Handbook on Corruption, Ethics and Integrity in Public Administration, Cheltenham, Edward Elgar Publishing.

Huberts, Leo y André van Montfort (2020b), "Integrity of Governance: Towards a System Approach", en C. Jurkiewicz, Global Corruption and Ethics Management, Londres, Rowan and Littlefield, pp. 184-193.

Huberts, Leo, Frank Anechiarico y Frédérique Six (2008), Local Integrity Systems: World Cities Fighting Corruption and Safeguarding Integrity, La Haya, BJu Legal Publishers.

Home Office (2018), Collection Anti-corruption, Londres, Gobierno del Reino Unido, disponible en: https://www.gov.uk/government/collections/anti-corruption [fecha de consulta: 23 de abril de 2021].

INTOSAI (2016), ISSAI 130, Viena. 
Jiménez Asensio, Rafael (2017), Cómo prevenir la corrupción: Integridad y transparencia, Madrid, La Catarata.

Lagos, Nicolás (2019), "Pseudomorfismo, cómo nos convencimos que Chile es un país corrupto: Herramientas para promover la integridad y la ética pública en Latinoamérica", en Centro Latinoamericano de Administración para el Desarrollo (CLAD) (ed.), Integridad yética en la función pública, Caracas, CLAD, pp. 41-100.

Lagos, Nicolás y Cristian Pliscoff (2020), "Implementando los sistemas de integridad en el sector público chileno: Reflexiones a partir del manual de la Organización para la Cooperación y el Desarrollo Económico sobre integridad pública”, Revista Chilena de la Administración del Estado, 3, pp. 35-48.

Macaulay, Michael, Chris Newman y Gary Hickey (2014), “Towards a Model of Local Integrity Systems: The Experiences of Local Government in Great Britain”, International Journal of Public Administration, 37(2), pp. 83-92.

Maesschalck, Jeroen (2004), "Approaches to Ethics Management in the Public Sector: A Proposed Extension of the Compliance-Integrity Continuum”, Public Integrity, 7(1), pp. 20-41.

Maesschalck, Jeroen (2019), "Facing the Dark Side”, en Carole Jurkiewicz (ed.), Global Corruption and Ethics Management: Translating Theory into Action, Londres, Rowman $\&$ Littlefield Publishers.

Maesschalck, Jeroen y János Bertók (2009), Towards a Sound Integrity Framework: Instruments, Processes, Structures and Conditions for Implementation, París, OCDE.

Menzel, Donald C. (2001), "Ethics Management in Public Organizations: What, Why, and How?" en Terry Cooper (dir.), Handbook of Administrative Ethics, Nueva York, Marcel Dekker, pp. 355-366.

OCDE (Organización para la Cooperación y el Desarrollo Económicos) (2020), OECD Public Integrity Handbook, París, OCDE.

OEA (Organización de los Estados Americanos) (1996), Convención Interamericana contra la corrupción, Caracas.

ONU (Organización de las Naciones Unidas) (2004), Convención de las Naciones Unidas contra la Corrupción, Nueva York.

Paine, Lynn (1994), "Managing for Organizational Integrity", Harvard Business Review, 72(2), pp. 106-117, Dor: 10.1177/105256299301700404.

Petersen Cortés, Germán (2020), "Un modelo de choques e interrupciones de reforma anticorrupción: Evidencia del caso mexicano", Gestión y Política Pública, XXIX(2), pp. 295-319.

Pliscoff, Cristian (2017), "Implementing the New Public Management: Problems and Challenges to Public Ethics: The Chilean Case", Convergencia, 24(73), pp. 1-22. 
PNUD (Programa de las Naciones Unidas para el Desarrollo) (2017), Sistemas de integridad $y$ códigos de ética, Santiago de Chile.

Pope, Jeremy (1996), National Integrity Systems: The TI Source Book, Transparency International (TI).

Quesada, Mónica García, Fernando Jiménez-Sánchez y Manuel Villoria (2013), "Building Local Integrity Systems in Southern Europe: The Case of Urban Local Corruption in Spain”, International Review of Administrative Sciences 79(4), pp. 618-637.

Rohr, John A. (1989), Ethics for Bureaucrats: An Essay on Law and Virtue, 2a. ed., Nueva York, Marcel Dekker.

Rose-Ackerman, Susan y Bonnie J. Palifka (2016), Corruption and Government: Causes, Consequences, and Reform, Cambridge, Cambridge University Press.

Sandoval Ballesteros, Irma Eréndira (2016), "Corrupción y desafíos organizacionales en un mundo de asociaciones público-privadas", Gestión y Política Pública, XXV(2), pp. 365-413.

Silva, Patricio (2016), "A Poor but Honest Country': Corruption and Probity in Chile", Journal of Developing Societies, 32(2), pp. 178-203.

SNa (Sistema Nacional Anticorrupción) (2021), ¿Qué hacemos?, Ciudad de México, sNA, disponible en: https://sna.org.mx/que-hacemos/ [fecha de consulta: 23 de abril de 2021].

Transparency International (2020), "What Is Corruption?” disponible en: https://www. transparency.org/what-is-corruption\#define [fecha de consulta: 8 de julio de 2020].

Unión Europea (2019), Directive (EU) 2019/1937 of the European Parliament and of the Council on the Protection of Persons Who Report Breaches of Union Law, 23 de octubre.

Villoria, Manuel (2014), "Integridad", Eunomía, Revista en Cultura de la Legalidad, 1, pp. 107-113.

\section{Fuentes normativas}

Ministerio de Hacienda (2017), Oficio ordinario núm. 1316, de 2017, que informa acciones vinculadas a la implementación del sistema de integridad en cada institución pública.

Dirección Nacional del Servicio Civil de Chile (2018a), Oficio ordinario núm. 335, de 2018, que solicita antecedentes vinculados a la implementación del sistema de integridad en cada institución pública.

Dirección Nacional del Servicio Civil de Chile (2018b), Oficio ordinario núm. 613 de 2018, que informa prórroga para la entrega de informe sobre proceso de implementación de los sistemas de integridad.

Ministerio Secretaría General de la Presidencia (2018), Oficio ordinario núm. 2305, de 2018, que recomienda acciones vinculadas a la implementación y difusión de los sistemas de integridad pública en los órganos de la Administración del Estado. 


\section{ANEXOS}

ANEXO 1. Oficios de construcción e implementación de sistemas de integridad Documento Ideasfuerza $\begin{array}{ll}\text { OFICIO ORD. } 1316 \text { del } & \text { Señala orientaciones relativas a la implementación de los sistemas de integridad } \\ \text { Ministerio de Hacienda, del } & \text { Pública durante el segundo semestre de } 2017\end{array}$ 27 de junio de 2017

Informa acciones vinculadas Define los objetivos de un sistema de integridad institucional: a implementación del sistema de integridad en cada institución pública

1. Contar con un código de ética institucional

2. Garantizar estrategias que incluyan, en el ámbito de la ética pública, el liderazgo del jefe/a superior de servicio

3. Contar con una estructura de funcionamiento

4. Velar por el cumplimiento de las disposiciones contenidas en el código de ética institucional

5. Desarrollar canales de información

6. Contar con canales de consulta y denuncias de inobservancias éticas

Sugiere la siguiente estructura formal para el diseño e implementación del sistema de integridad:

1. Jefe/a superior de servicio (líder y referente ético)

2. Asesores/as técnicos/as (apoyo experto para la toma de decisiones del jefe/a de servicio)

3. Coordinador/a de integridad (implementa acciones y herramientas para la cultura ética)

4. Comité de integridad (representa a los funcionarios y propone estrategias éticas)

5. Plataforma de gestión de integridad (apoyo tecnológico en la comunicación del sistema)

OFICIO ORD. 0335 de la

Dirección Nacional del

Servicio Civil del 30 de enero de 2018

Solicita antecedentes vinculados a la implementación del sistema de integridad en cada institución pública
Solicita generar un informe que contenga:

I. Implementación infraestructura del sistema:

1. Resolución que crea el comité de integridad

2. Resolución que designa a los asesores técnicos

3. Resolución que nombra al coordinador de integridad

4. Dirección web institucional con el código de ética en un lugar relevante

5. Dirección web de la intranet especificando el apartado sistema de integridad y código de ética

II. Construcción de la plataforma tecnológica:

1. La estructura del sistema de integridad

2. Canal de denuncia y sugerencia

3. Los procesos de capacitación y difusión

III. Capacitación y difusión:

1. Cronograma de capacitaciones realizadas

2. Contenido de la capacitación

3. Cronograma del proceso de difusión realizada

4. Contenido de la difusión 


\section{ANEXO 1. Oficios de construcción e implementación de sistemas de integridad (continuación)}

\begin{tabular}{ll}
\multicolumn{1}{c}{ Documento } & \multicolumn{1}{c}{ Ideas fuerza } \\
\hline OFICIO ORD. 613 de la & Informa extensión de plazo hasta el 30 de julio de 2018 para el envío del informe \\
Dirección Nacional del & sobre implementación de sistemas de integridad en instituciones públicas. \\
Servicio Civil del 27 de & \\
marzo de 2018. & \\
Informa prórroga. & \\
\hline
\end{tabular}

Fuente: Elaboración propia a partir de Ministerio de Hacienda (2017), Dirección Nacional del Servicio Civil de Chile (2018a, 2018b) y Ministerio Secretaría General de la Presidencia (2018).

\section{ANEXO 2. Solicitud de información generada para el levantamiento de información}

1 Resolución que crea el comité de integridad

2 Fecha de creación del comité

3 Número de reuniones en que ha sesionado desde la creación hasta el 10 de diciembre de 2019

4 Resolución que designa a los asesores técnicos

5 Resolución que nombra al coordinador de integridad

6 Imagen de la web institucional con el código de ética en un lugar relevante

7 Imagen de la web de la intranet especificando el apartado sistema de integridad y código de ética

8 Medio de verificación de la existencia de un canal de denuncias o consultas

9 ¿El canal de consultas y denuncias permite el ingreso de solicitudes anónimas?

10 ¿El canal de consultas y denuncias permite el ingreso de solicitudes de personas externas a la institución?

11 Fecha de inicio del canal de consultas y denuncias

12 Número de consultas recibidas desde el inicio hasta el 10 de diciembre de 2019 y porcentaje realizado bajo anonimato

13 Número de denuncias recibidas desde el inicio hasta el 10 de diciembre de 2019 y porcentaje realizado bajo anonimato

Fuente: Elaboración propia. 
ANEXO 3. Clústeres institucionales identificados

\begin{tabular}{|c|c|c|c|c|c|}
\hline Clúster & Núm. & Institución & Cluster & Núm. & Institución \\
\hline A & 1 & $\begin{array}{l}\text { Agencia de Promoción a la Inversión } \\
\text { Extranjera }\end{array}$ & B & 5 & $\begin{array}{l}\text { Caja de Previsión de la Defensa } \\
\text { Nacional }\end{array}$ \\
\hline A & 2 & Servicio de Salud de Antofagasta & B & 6 & Comisión Nacional de Energía \\
\hline A & 3 & CRS Cordillera Oriente & B & 7 & Consejo Nacional de Educación \\
\hline A & 4 & Consejo de Defensa del Estado & B & 8 & Dirección General de Concesiones \\
\hline A & 5 & Consejo de Rectores & B & 9 & Corporación de Fomento \\
\hline A & 6 & Dipreca & B & 10 & CRS Maipú \\
\hline A & 7 & Gore Tarapacá & B & 11 & Dirección de Contabilidad y Finanzas \\
\hline A & 8 & Hospital Padre Hurtado & & & MOP \\
\hline \multirow[t]{2}{*}{ A } & \multirow[t]{2}{*}{9} & \multirow{2}{*}{$\begin{array}{l}\text { Oficina de Estudios y Políticas } \\
\text { Agrarias }\end{array}$} & B & 12 & Corporación de Desarrollo Indígena \\
\hline & & & B & 13 & Defensoría Penal Pública \\
\hline \multirow[t]{2}{*}{ A } & \multirow[t]{2}{*}{10} & \multirow{2}{*}{$\begin{array}{l}\text { Servicio de Registro Civil e } \\
\text { Identificación }\end{array}$} & B & 14 & Presidencia de la República \\
\hline & & & B & 15 & Dirección de Arquitectura MOP \\
\hline A & 11 & Servicio de Salud de Arauco & B & 16 & Dirección de Crédito Prendario \\
\hline A & 12 & Servicio Nacional del Consumidor & B & 17 & Dirección de Aeropuertos MOP \\
\hline A & 13 & Servicio de Salud de Araucanía Sur & B & 18 & Dirección de Planificación MOP \\
\hline A & 14 & Servicio de Salud de Chiloé & B & 19 & Dirección General de Aguas MOP \\
\hline A & 15 & Servicio de Salud de Coquimbo & B & 20 & Dirección de Fronteras y Límites \\
\hline A & 16 & Servicio de Salud Metropolitano Central & B & 21 & Dirección Nacional del Servicio Civil \\
\hline A & 17 & Servicio de Salud de O'Higgins & B & 22 & Fiscalía MOP \\
\hline A & 18 & Serviu Maule & B & 23 & Fiscalía Nacional Económica \\
\hline A & 19 & Serviu Los Lagos & B & 24 & Fondo Nacional de Salud \\
\hline A & 20 & $\begin{array}{l}\text { Servicio de Salud de Viña del Mar- } \\
\text { Quillota }\end{array}$ & B & 25 & $\begin{array}{l}\text { Fondo de Solidaridad e Inversión } \\
\text { Social }\end{array}$ \\
\hline A & 21 & Subsecretaría de Educación & B & 26 & Gendarmería Chile \\
\hline A & 22 & Subsecretaría de Energía & B & 27 & Intendencia Maule \\
\hline A & 23 & Subsecretaría Segegob & B & 28 & Intendencia Arica y Parinacota \\
\hline A & 24 & Subsecretaría de Salud Pública & B & 29 & Intendencia Tarapacá \\
\hline A & 25 & Superintendencia de Salud & B & 30 & Intendencia Antofagasta \\
\hline B & 1 & Agenda de Calidad de la Educación & B & 31 & Intendencia Araucanía \\
\hline B & 2 & Gore Atacama & B & 32 & Intendencia Metropolitana \\
\hline B & 3 & Subsecretaría Bienes Nacionales & B & 33 & Intendencia O'Higgins \\
\hline B & 4 & Subsecretaría de la Mujer & B & 34 & Intendencia de Aysen \\
\hline
\end{tabular}


ANEXO 3. Clústeres institucionales identificados (continuación)

\begin{tabular}{|c|c|c|c|c|c|}
\hline Cluster & Núm. & Institución & Clúster & Núm. & Institución \\
\hline B & 35 & Intendencia Magallanes & B & 62 & Servicio de Vivienda y Urbanismo \\
\hline B & 36 & Intendencia Los Ríos & & & Antofagasta \\
\hline B & 37 & Gobierno Regional de Antofagasta & B & 63 & $\begin{array}{l}\text { Servicio de Vivienda y Urbanismo } \\
\text { Araucanía }\end{array}$ \\
\hline B & 38 & Gobierno Regional de Araucanía & B & 64 & Servicio de Vivienday Urbanismo Aysen \\
\hline $\begin{array}{l}B \\
B\end{array}$ & $\begin{array}{l}39 \\
40\end{array}$ & $\begin{array}{l}\text { Gobierno Regional de Aysen } \\
\text { Gobierno Regional del Biobio }\end{array}$ & B & 65 & $\begin{array}{l}\text { Servicio de Vivienda y Urbanismo } \\
\text { Coquimbo }\end{array}$ \\
\hline $\begin{array}{l}B \\
B\end{array}$ & $\begin{array}{l}41 \\
42\end{array}$ & $\begin{array}{l}\text { Gobierno Regional de Los Ríos } \\
\text { Gobierno Regional Metropolitano }\end{array}$ & B & 66 & $\begin{array}{l}\text { Servicio de Vivienda y Urbanismo } \\
\text { Los Ríos }\end{array}$ \\
\hline$B$ & 43 & Gobierno Regional de O'Higgins & B & 67 & $\begin{array}{l}\text { Servicio de Vivienda y Urbanismo } \\
\text { Biobio }\end{array}$ \\
\hline $\begin{array}{l}B \\
B\end{array}$ & $\begin{array}{l}44 \\
45\end{array}$ & $\begin{array}{l}\text { Instituto Antártico Chileno } \\
\text { Instituto Nacional de Desarrollo }\end{array}$ & B & 68 & $\begin{array}{l}\text { Servicio de Vivienda y Urbanismo } \\
\text { Metropolitano }\end{array}$ \\
\hline B & 46 & $\begin{array}{l}\text { Agropecuario } \\
\text { Instituto Nacional de Hidráulica }\end{array}$ & B & 69 & $\begin{array}{l}\text { Servicio de Vivienda y Urbanismo } \\
\text { Tarapacá }\end{array}$ \\
\hline B & 47 & Instituto Nacional de la Juventud & B & 70 & Subsecretaría de Agricultura \\
\hline B & 48 & $\begin{array}{l}\text { Institucional Nacional de Propiedad } \\
\text { Intelectual }\end{array}$ & B & 71 & Subsecretaría de Defensa \\
\hline B & 49 & Junta Nacional de Aeronáutica Civil & B & 72 & $\begin{array}{l}\text { Subsecretaría de Desarrollo Regional } \\
\text { y Administrativo }\end{array}$ \\
\hline B & 50 & $\begin{array}{l}\text { Junta Nacional de Auxilio Escolary } \\
\text { Becas }\end{array}$ & B & 73 & Subsecretaría de Pesca y Acuicultura \\
\hline B & 51 & $\begin{array}{l}\text { Servicio Nacional de la Mujer y la } \\
\text { Equidad de Género }\end{array}$ & $\begin{array}{l}B \\
B\end{array}$ & $\begin{array}{l}74 \\
75\end{array}$ & $\begin{array}{l}\text { Subsecretaría de Previsión Social } \\
\text { Subsecretaría de Transportes }\end{array}$ \\
\hline B & 52 & Servicio Agrícola y Ganadero & B & 76 & $\begin{array}{l}\text { Subsecretaría de Vivienda y } \\
\text { Urbanismo }\end{array}$ \\
\hline B & 53 & $\begin{array}{l}\text { Servicio Nacional de Capacitación } \\
\text { y Empleo }\end{array}$ & B & 77 & Subsecretaría del Deporte \\
\hline B & 54 & Servicio de Salud de Arica & B & 78 & Subsecretaría del Trabajo \\
\hline$B$ & 55 & Servicio Médico Legal & B & 79 & $\begin{array}{l}\text { Subsecretaría de Economía y } \\
\text { Empresas de Menor Tamaño }\end{array}$ \\
\hline B & 56 & Servicio Nacional del Adulto Mayor & B & 80 & Subsecretaría de Evaluación Social \\
\hline B & 57 & Servicio Nacional de Discapacidad & B & 81 & Subsecretaría de Fuerzas Armadas \\
\hline B & 58 & Servicio Nacional de Turismo & B & 82 & Subsecretaría de Medio Ambiente \\
\hline B & 59 & Servicio Nacional de Menores & B & 83 & Subsecretaría de Minería \\
\hline B & 60 & $\begin{array}{l}\text { Servicio de Salud Metropolitano } \\
\text { Occidente }\end{array}$ & B & 84 & Subsecretaría de Obras Públicas \\
\hline B & 61 & $\begin{array}{l}\text { Servicio de Salud Metropolitano } \\
\text { Oriente }\end{array}$ & $\begin{array}{l}\text { B } \\
B\end{array}$ & $\begin{array}{l}85 \\
86\end{array}$ & $\begin{array}{l}\text { Subsecretaría de Prevención del Delito } \\
\text { Subsecretaría de Relaciones } \\
\text { Exteriores }\end{array}$ \\
\hline
\end{tabular}


ANEXO 3. Clústeres institucionales identificados (continuación)

\begin{tabular}{|c|c|c|c|c|c|}
\hline Clúster & Núm. & Institución & Cluster & Núm. & Institución \\
\hline B & 87 & Subsecretaría de Turismo & C & 22 & Servicio Nacional de Aduanas \\
\hline B & 88 & Comisión para el Mercado Financiero & $\mathrm{C}$ & 23 & Servicio de Salud de lquique \\
\hline B & 89 & Superintendencia de Educación & $\mathrm{C}$ & 24 & Servicio de Salud Metropolitano Norte \\
\hline B & 90 & $\begin{array}{l}\text { Superintendencia de Medio } \\
\text { Ambiente }\end{array}$ & C & 25 & $\begin{array}{l}\text { Servicio de Vivienda y Urbanismo } \\
\text { Magallanes }\end{array}$ \\
\hline B & 91 & $\begin{array}{l}\text { Superintendencia de Servicios } \\
\text { Sanitarios }\end{array}$ & C & 26 & $\begin{array}{l}\text { Servicio de Vivienda y Urbanismo } \\
\text { O'Higgins }\end{array}$ \\
\hline B & 92 & Servicio de Salud de Talcahuano & C & 27 & Servicio de Vivienda y Urbanismo \\
\hline \multirow[t]{2}{*}{ B } & \multirow[t]{2}{*}{93} & \multirow{2}{*}{ Unidad de Análisis Financiero } & & & \\
\hline & & & C & 28 & Subsecretaría de Servicios Sociales \\
\hline C & 1 & Servicio de Salud de Bio Bio & \multirow[t]{2}{*}{ C } & \multirow[t]{2}{*}{29} & \multirow{2}{*}{$\begin{array}{l}\text { Superintendencia de Casinos de } \\
\text { Juego }\end{array}$} \\
\hline C & 2 & Central Nacional de Abastecimiento & & & \\
\hline C & 3 & Cochilco & C & 30 & Superintendencia de Insolvencia y \\
\hline$C$ & 4 & Comisión Chilena de Energía Nuclear & & & \\
\hline C & 5 & Comisión Nacional de Riego & C & 31 & Superintendencia de Pensiones \\
\hline \multirow{2}{*}{ C } & \multirow[t]{2}{*}{6} & \multirow{2}{*}{$\begin{array}{l}\text { Consejo Nacional de Investigación } \\
\text { Científica y Tecnología }\end{array}$} & C & 32 & Servicio de Salud Metropolitano Sur \\
\hline & & & C & 33 & Subsecretaría de Justicia \\
\hline \multirow[t]{2}{*}{ C } & \multirow[t]{2}{*}{7} & \multirow{2}{*}{$\begin{array}{l}\text { Servicio Nacional del Patrimonio } \\
\text { Cultural }\end{array}$} & C & 34 & Superintendencia de Seguridad Social \\
\hline & & & $\mathrm{C}$ & 35 & Subsecretaría de Obras Públicas \\
\hline C & 8 & Dirección General de Obras Públicas & $\mathrm{C}$ & 36 & Servicio de Salud del Bio Bio \\
\hline $\mathrm{C}$ & 9 & Dirección de Obras Portuarias & \multirow[t]{2}{*}{ C } & \multirow[t]{2}{*}{37} & \multirow{2}{*}{$\begin{array}{l}\text { Consejo Nacional de la Cultura y las } \\
\text { Artes }\end{array}$} \\
\hline C & 10 & Gobierno Regional de Coquimbo & & & \\
\hline $\mathrm{C}$ & 11 & Gobierno Regional de Los Lagos & C & 38 & Subsecretaría de Hacienda \\
\hline $\mathrm{C}$ & 12 & Gobierno Regional de Valparaíso & \multirow[t]{2}{*}{ C } & \multirow[t]{2}{*}{39} & \multirow{2}{*}{$\begin{array}{l}\text { Subsecretaría de Educación } \\
\text { Parvularia }\end{array}$} \\
\hline C & 13 & Instituto de Previsión Social & & & \\
\hline C & 14 & Instituto de Seguridad Laboral & \multirow[t]{2}{*}{$\mathrm{D}$} & \multirow[t]{2}{*}{1} & \multirow{2}{*}{$\begin{array}{l}\text { Dirección de Compras Públicas- } \\
\text { Chilecompra }\end{array}$} \\
\hline C & 15 & Junta Nacional de Jardines Infantiles & & & \\
\hline \multirow[t]{2}{*}{ C } & \multirow[t]{2}{*}{16} & \multirow{2}{*}{$\begin{array}{l}\text { Subsecretaría General de la } \\
\text { Presidencia }\end{array}$} & D & 2 & Instituto Nacional de Deportes \\
\hline & & & $\mathrm{D}$ & 3 & Instituto Nacional de Estadísticas \\
\hline C & 17 & Oficina Nacional de Emergencia & \multirow[t]{2}{*}{ D } & \multirow[t]{2}{*}{4} & \multirow{2}{*}{$\begin{array}{l}\text { Servicio de Salud Metropolitano Sur } \\
\text { Oriente }\end{array}$} \\
\hline C & 18 & Parque Metropolitano & & & \\
\hline C & 19 & Servicio de Cooperación Técnica & $\mathrm{D}$ & 5 & Servicio de Salud de Reloncaví \\
\hline C & 20 & Sistema de Evaluación Ambiental & $\mathrm{D}$ & 6 & Servicio de Impuestos Internos \\
\hline C & 21 & Servicio de Salud Araucanía Norte & $\mathrm{D}$ & 7 & Tesorería General de la República \\
\hline
\end{tabular}

Fuente: Elaboración propia. 
ANEXO 4. Instituciones y encargados seleccionados para etapa de entrevistas

\begin{tabular}{|c|c|}
\hline Institución & Participante \\
\hline Dirección de Compras y Contratación Pública & $\begin{array}{l}\text { Viviana Mora, coordinadora de Integridad y jefa del } \\
\text { Observatorio Chile Compra }\end{array}$ \\
\hline Instituto Nacional de Deportes (IND) & $\begin{array}{l}\text { Jorge Vergara, coordinador de Integridad y jefe de Planificación } \\
\text { y Control de Gestión }\end{array}$ \\
\hline $\begin{array}{l}\text { Servicio de Salud Metropolitano Oriente } \\
\text { (SSMSO) }\end{array}$ & $\begin{array}{l}\text { Cinthia León, coordinadora de Integridad y encargada del } \\
\text { sistema de prevención }\end{array}$ \\
\hline Tesorería General de la República (TGR) & $\begin{array}{l}\text { Adrián Fuentes, coordinador de Integridad y jefe de unidad de } \\
\text { Cumplimiento Regulatorio }\end{array}$ \\
\hline Junta Nacional de Jardines Infantiles (Junji) & Macarena Muñoz, coordinadora de Integridad \\
\hline $\begin{array}{l}\text { Junta Nacional de Auxilio Escolary Becas } \\
\text { (Junaeb) }\end{array}$ & $\begin{array}{l}\text { Daniela Cajas, coordinadora de Integridad y de jefa } \\
\text { Procedimientos Disciplinarios }\end{array}$ \\
\hline Ministerio de Obras Públicas & Carolina Pedemonte, encargada de Integridad Ministerial \\
\hline Servicio Nacional de Aduanas & Sin respuesta \\
\hline Servicio de Salud de Reloncaví & Sin respuesta \\
\hline Servicio de Impuestos Internos (SII) & Sin respuesta \\
\hline Instituto Nacional de Estadísticas (INE) & Sin Respuesta \\
\hline
\end{tabular}

Fuente: Elaboración propia. 


\section{Anexo 5. Pauta de entrevista}

\section{Preguntas}

\section{Contexto}

1.1 ¿Cuál es su cargo actual y cuál es su vinculación con las temáticas de integridad y ética en la organización?

1.2 ¿Cuál fue su participación en el proceso de elaboración del código de ética desarrollado el año 2016 ?

2. Funcionamiento del sistema de integridad

2.1 ¿Nos podría describir cuál es la estructura formal del sistema?

2.2 ¿Cómo opera el sistema en la práctica? ¿Cuáles son los roles que participan mayormente en la ejecución del sistema?

2.3 ¿Cómo es el funcionamiento formal y habitual del Comité de Integridad?

2.4 ¿Cómo funciona el canal de consultas y denuncias?

2.5 ¿Qué dificultades prácticas se tienen?

2.6 En una escala de 1 a 10, donde 1 es comienzo de la implementación y 10 totalmente implementado, ¿dónde ubicaría la implicación del sistema en su organización?

3. Factores de éxito

3.1 ¿Qué factores, elementos o situaciones han favorecido la implementación del sistema en su organización?

4. Rol de distintos actores

4.1 ¿Qué percepción tienen los funcionarios y las asociaciones de funcionarios del sistema?

4.2 ¿Qué importancia le asignan al rol del directivo de la institución en la implementación del sistema?

4.2 ¿Qué rol jugó la DNSC en la implementación inicial del sistema?

4.3 ¿Qué rol juega actualmente la DNSC en la implementación del sistema?

4.4 ¿Conoce la realidad de otras organizaciones en la implementación de estas iniciativas?

4.5 ¿Qué recomendaciones daría a otras instituciones que no han logrado implementar óptimamente estas iniciativas?

Fuente: Elaboración propia. 
Cristian Pliscoff Varas, administrador público y magíster en Ciencia Política por la Universidad de Chile; maestro en Administración y Política Pública por la London School of Economics, y doctor en Administración Pública por University of Southern California. Profesor asociado de la Universidad de Chile. Ex presidente de la Red Interamericana de Educación en Administración Pública (2016-2018), consultor nacional e internacional en materia de modernización de la gestión pública y gestión de personas en el sector público. Sus líneas de investigación y publicaciones se refieren a los temas de ética y probidad en el sector público, modernización de la gestión pública y educación en administración pública. Actualmente es el coordinador del magíster en Gobierno y Gerencia Pública de la Universidad de Chile.

Nicolás Lagos Machuca, administrador público, magíster en Gobierno y Gerencia Pública y profesor de la Escuela de Gobierno y Gestión Pública de la Universidad de Chile. En 2017 ganó el concurso sobre "Integridad y ética en la función pública" del Centro Latinoamericano de Administración para el Desarrollo (CLAD). Desde 2015 ha trabajado en la implementación en Chile de la Convención de las Naciones Unidas contra la Corrupción. Es miembro del Gabinete del Contralor General, coordinador de los proyectos asociados a la lucha contra la corrupción y la promoción de la integridad. 